\title{
Extreme hyponatraemia due to primary polydipsia and quetiapine-induced SIAD
}

\author{
Darran Mc Donald(101,2, Tara Mc Donnell[1, Rachel Katherine Crowley2,3 and \\ Elizabeth Brosnan ${ }^{1}$
}

1'Department of Medicine, Mayo University Hospital, Mayo, Ireland, 2Department of Endocrinology, St Vincent's University Hospital, Dublin, Ireland, and ${ }^{3}$ School of Medicine, University College Dublin, Dublin, Ireland
Correspondence

should be addressed

to D Mc Donald

Email

darranmcdonald@gmail.com

\section{Summary}

Hyponatraemia is the most common electrolyte disturbance in hospitalised patients and is associated with numerous adverse outcomes. Patients with schizophrenia are particularly susceptible to hyponatraemia, in part due to the close association between this condition and primary polydipsia. We report the case of a 57-year-old woman with schizophrenia and primary polydipsia who was receiving inpatient psychiatric care. She became increasingly confused, had multiple episodes of vomiting, and collapsed 1 week after being commenced on quetiapine $300 \mathrm{mg}$. On examination, she was hypertensive and her Glasgow coma scale was nine. She had a fixed gaze palsy and a rigid, flexed posture. Investigations revealed extreme hyponatraemia with a serum sodium of $97 \mathrm{mmol} / \mathrm{L}$. A CT brain demonstrated diffused cerebral oedema with sulcal and ventricular effacement. A urine sodium and serum osmolality were consistent with SIAD, which was stimulated by the introduction of quetiapine. The antidiuretic effect of vasopressin limited the kidney's ability to excrete free water in response to the patients' excessive water intake, resulting in extreme, dilutional hyponatraemia. The patient was treated with two $100 \mathrm{~mL}$ boluses of hypertonic $3 \%$ saline but deteriorated further and required intubation. She had a complicated ICU course but went on to make a full neurological recovery. This is one of the lowest sodium levels attributed to primary polydipsia or second-generation antipsychotics reported in the literature.

\section{Learning points:}

- The combination of primary polydipsia and SIAD can lead to a life-threatening, extreme hyponatraemia.

- SIAD is an uncommon side effect of second-generation anti-psychotics.

- Serum sodium should be monitored in patients with primary polydipsia when commencing or adjusting psychotropic medications.

- Symptomatic hyponatraemia is a medical emergency that requires treatment with boluses of hypertonic $3 \%$ saline.

- A serum sodium of less than $105 \mathrm{mmol} / \mathrm{L}$ is associated with an increased risk of osmotic demyelination syndrome, therefore the correction should not exceed $8 \mathrm{mmol} / \mathrm{L}$ over $24 \mathrm{~h}$.

\section{Background}

Hyponatraemia is the most common electrolyte abnormality in hospitalised patients. It is associated with adverse outcomes including prolonged hospital admissions and increased mortality (1). The severity of hyponatraemia can be classified as mild (130-134 mmol/L), moderate
(125-129 mmol/L) or profound (<125 mmol/L) (1). Most cases of hyponatraemia are mild. It has been demonstrated that even mild cases of chronic hyponatremia can impair attention, gait and increases the risk of osteoporosis (2). Additionally, a minority can present with severe, 
symptomatic hyponatraemia. Symptoms include confusion, vomiting, altered consciousness, seizures and coma. Symptomatic hyponatraemia is caused by a rapid reduction in the serum sodium within $48 \mathrm{~h}$. Fluid moves from the hypo-osmolar intravascular space, across the blood-brain barrier and into the intracellular space leading to cerebral oedema. This increases intracranial pressure and can potentially lead to tonsillar herniation and death. Symptomatic hyponatremia is a medical emergency and requires urgent treatment with boluses of hypertonic $3 \%$ saline to relieve cerebral oedema and prevent progression to herniation (1). Syndrome of inappropriate antidiuresis (SIAD) is the most frequent underlying cause of hyponatraemia in hospitalised patients. SIAD can be caused by a wide variety of conditions and medications.

Patients with schizophrenia are particularly susceptible to hyponatraemia due to the frequent use of SIAD-inducing drugs. Another contributory factor is the close association between schizophrenia and primary polydipsia (PP), which occurs in approximately 10\% of patients with schizophrenia (3). PP is characterised by altered thirst leading to excessive fluid intake and the production of large volumes of dilute urine, exceeding three litres per day, having excluded secondary cause of polydipsia (1) This report describes a rare case of extreme hyponatraemia due to primary polydipsia and quetiapineinduced SIAD in a patient with schizophrenia. This is one of the lowest reported serum sodium levels ever reported in a patient with PP or attributed to second-generation anti-psychotics (SGA). The radiological images, captured after the patients collapse demonstrating diffuse cerebral oedema highlight why symptomatic hyponatremia is a potential life-threatening medical emergency.

\section{Case presentation}

A 57-year-old Caucasian woman with schizophrenia was admitted to a psychiatric unit following an episode of deliberate self-harm. She also had a history of type 2 diabetes mellitus and hypothyroidism. Current medications included aspirin $75 \mathrm{mg}$, metformin $1 \mathrm{~g}$ twice daily, risperidone $4 \mathrm{mg}$ twice daily and thyroxine $175 \mu$ g. Nursing staff noted that the patient drank water excessively and stopped her drinking water directly from taps on several occasions. No formal measurement of fluid intake was documented, and there was no known history of polydipsia. Four weeks after admission, her antipsychotic regimen was changed from risperidone $4 \mathrm{mg}$ twice daily to quetiapine $300 \mathrm{mg}$ once daily. One week after this change, the patient became increasingly confused. She started acting bizarrely and took repeated showers while fully clothed. Several hours later, she vomited, collapsed and became poorly responsive. On examination, she was clinically euvolaemic but hypertensive with a blood pressure of 180/110 mmHg. Glasgow Coma Scale (GCS) was 9. She had a fixed gaze palsy, and her pupils were poorly reactive to light. Her limbs were rigid with a flexed posture and she was only able to make incomprehensible sounds.

\section{Investigation}

Investigations revealed extreme hyponatraemia with plasma sodium $\left(\mathrm{Na}^{+}\right)$of $97 \mathrm{mmol} / \mathrm{L}$, serum osmolality (Osm) $205 \mathrm{mmol} / \mathrm{L}$, urea $2.8 \mathrm{mmol} / \mathrm{L}$ and creatinine 46 $\mu \mathrm{mol} / \mathrm{L}$ (Table 1). Her serum $\mathrm{Na}^{+}$was $134 \mathrm{mmol} / \mathrm{L}$ upon admission to the psychiatric unit, 5 weeks earlier. She had no prior history of hyponatremia. Serum glucose was 5.8 $\mathrm{mmol} / \mathrm{L}$. A low urea of $2.8 \mathrm{mmol} / \mathrm{L}$ and haematocrit of $0.266 \mathrm{~L} / \mathrm{L}$ were consistent with the history of polydipsia. Her urine $\mathrm{Na}^{+}$and urine Osm were $52 \mathrm{mmol} / \mathrm{L}$ and $178 \mathrm{mmol} / \mathrm{L}$, respectively. The patient underwent an emergency CT scan. It revealed diffuse cerebral oedema, with loss of grey-white matter differentiation and sulcal and ventricular effacement (Fig. 1). These findings raised concern for imminent tonsillar herniation.

Thyroid function tests (TFTs) confirmed the patient was euthyroid on thyroxine replacement with a TSH of 0.88 $\mathrm{mmol} / \mathrm{L}(0.77-1.03)$ and fT4 $22.6 \mathrm{pmol} / \mathrm{L}$ (12-24). A 9:00 $\mathrm{h}$ cortisol of $675 \mathrm{nmol} / \mathrm{L}$ excluded adrenal insufficiency. The urine Osm of $178 \mathrm{mmol} / \mathrm{L}$ confirmed the presence of vasopressin (AVP) as there was a partial concentration of urine. This was inappropriate given the low serum osmolality and attributed to a quetiapine-induced SIAD. We suspect the patient drank large quantities of water while she showered repeatedly prior to her collapse. SIAD limited the patient's ability to excrete free water, leading to extreme dilutional hyponatraemia.

Table 1 Serum and urine biochemistry profile.

\begin{tabular}{|c|c|c|}
\hline Laboratory parameter & Result & Reference range \\
\hline Serum $\mathrm{Na}^{+}, \mathrm{mmol} / \mathrm{L}$ & 97 & $133-146$ \\
\hline Serum osmolality, mmol/L & 205 & $285-295$ \\
\hline Urea, $\mathrm{mmol} / \mathrm{L}$ & 2.8 & $2.5-7.8$ \\
\hline Creatinine, $\mathrm{mol} / \mathrm{L}$ & 46 & 59-104 \\
\hline Glucose, $\mathrm{mmol} / \mathrm{L}$ & 5.8 & \\
\hline Haematocrit, L/L & 0.266 & $0.34-0.46$ \\
\hline Urine $\mathrm{Na}^{+}, \mathrm{mmol} / \mathrm{L}$ & 52 & \\
\hline Urine osmolality, $\mathrm{mmol} / \mathrm{L}$ & 178 & \\
\hline $\mathrm{TSH}, \mathrm{mmol} / \mathrm{L}$ & 0.88 & $0.77-1.03$ \\
\hline fT4, pmol/L & 22.6 & $12-24$ \\
\hline 9am cortisol, $\mathrm{nmol} / \mathrm{L}$ & 675 & $>420$ \\
\hline
\end{tabular}




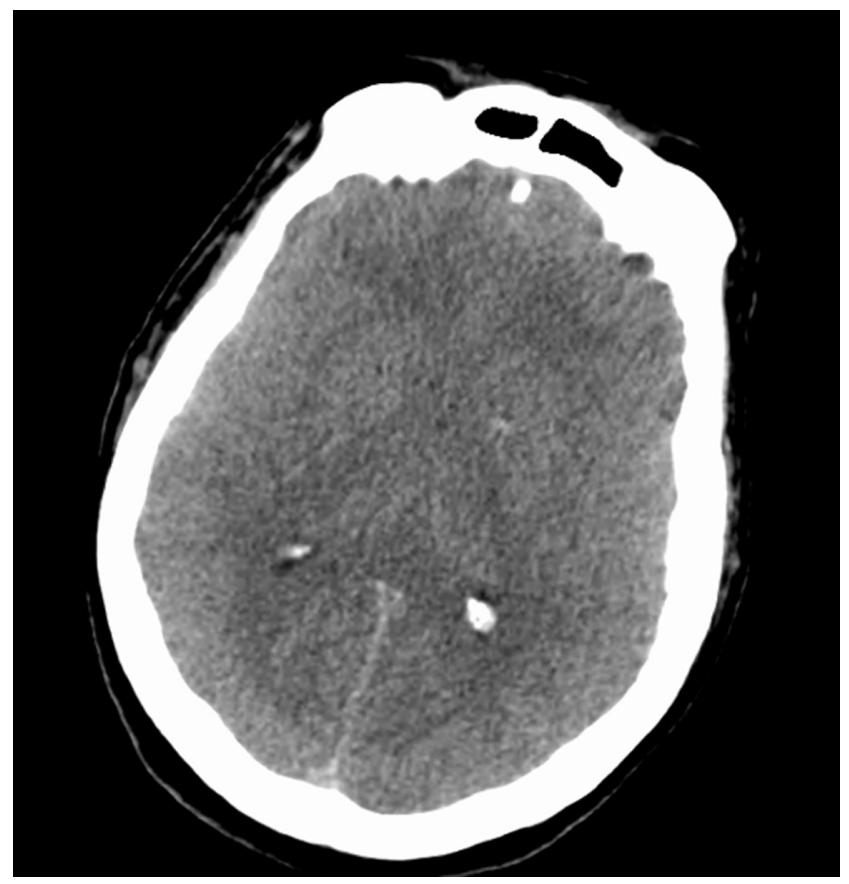

Figure $1 \mathrm{CT}$ brain immediately after the collapse showing loss of grey-white matter differentiation and sulcal and ventricular effacement, indicating diffuse cerebral oedema.

\section{Treatment}

The patient received two $100 \mathrm{~mL}$ i.v. boluses of hypertonic $3 \%$ saline, each administered over $10 \mathrm{~min}$. There was no neurological improvement despite the serum $\mathrm{Na}^{+}$ increasing to $101 \mathrm{mmol} / \mathrm{L}$. She was then transferred to the ICU for close observation. The $\mathrm{Na}^{+}$correction limit was set at $8 \mathrm{mmol} / \mathrm{L}$ over $24 \mathrm{~h}$ as the patient was at high risk of developing osmotic demyelination syndrome (ODS). By $5 \mathrm{~h}$, the patient's urine output exceeded 400 $\mathrm{mL} / \mathrm{h}$, and serum $\mathrm{Na}^{+}$had increased to $104 \mathrm{mmol} / \mathrm{L}$. This aquaresis was caused by a reduction in circulating AVP after stopping quetiapine. Dextrose 5\% was administered to replace the free water excreted by the kidneys in an attempt to prevent further increases in serum $\mathrm{Na}^{+}$. Despite treatment with dextrose $5 \%$, the serum $\mathrm{Na}^{+}$increased to $109 \mathrm{mmol} / \mathrm{L}$ after $24 \mathrm{~h}$, exceeding the correction limit of $105 \mathrm{mmol} / \mathrm{L}$. The patient then developed an aspiration pneumonia and deteriorated rapidly. Her GCS fell to six and she developed significant respiratory distress, ultimately requiring intubation. A repeat CT brain showed a significant reduction in cerebral oedema (Fig. 2). The patient intermittently received 5\% dextrose over the next $72 \mathrm{~h}$ to prevent further rapid increases in the serum $\mathrm{Na}^{+}$. $\mathrm{Na}^{+}$levels increased to $119 \mathrm{mmol} / \mathrm{L}$ at $48 \mathrm{~h}, 126 \mathrm{mmol} / \mathrm{L}$ at $72 \mathrm{~h}$ and $134 \mathrm{mmol} / \mathrm{L}$ at $96 \mathrm{~h}$.

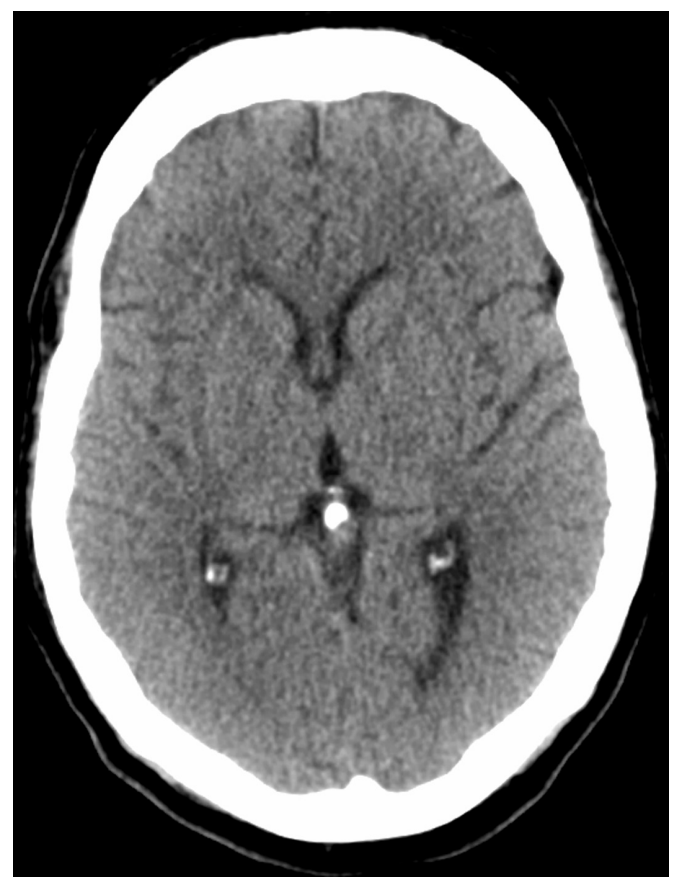

Figure 2 Interval CT brain performed 24 hrs following the collapse. It showed a resolution of the cererbal oedema following treatment for hyponatraemia.

\section{Outcome and follow-up}

The patient had a prolonged 29-day ICU admission, which was complicated by two ventilator-associated pneumonias. She underwent a MRI brain 2 weeks into her ICU admission which did not reveal any evidence of ODS. The patient went on to make a full recovery and was transferred back to the psychiatric unit.

She was recommenced on risperidone and placed on a $2 \mathrm{~L}$ fluid restriction. Her sodium remained in the range 134-138 $\mathrm{mmol} / \mathrm{L}$ throughout the remainder of her hospital admission. Upon discharge, the patient was counselled to avoid excessive fluid intake. Her sodium at her subsequent follow-up appointment was $136 \mathrm{mmol} / \mathrm{L}$.

\section{Discussion}

We describe a case of extreme, symptomatichyponatraemia, secondary to PP and quetiapine-induced SIAD in a patient with schizophrenia. It is our clinical observation that cases such as this one, with symptomatic hyponatraemia due to particularly low serum sodium, often have multifactorial aetiologies. Our case is noteworthy for the degree of hyponatraemia and that we captured radiological images of the resulting cerebral oedema. Furthermore, the patient made a complete recovery despite the extreme hyponatraemia. This is in contrast to the majority of 
published cases which frequently describe fatal outcomes related to hyponatraemia or complications of treatment including ODS. We attribute this patient's outcome to the recognition of symptomatic hyponatraemia as a medical emergency resulting in prompt medical intervention. Current guidelines on the management of symptomatic hyponatraemia advise administering 1-3 boluses of 100 $\mathrm{mL}$ hypertonic 3\% saline over $10 \mathrm{~min}$ (4). It should be noted that European guidelines differ somewhat, advising 1-2 boluses of $150 \mathrm{~mL}$ hypertonic 3\% saline over $20 \mathrm{~min}$ (1). It has been shown that boluses of hypertonic 3\% saline increase $\mathrm{Na}^{+}$more rapidly than infusions, without increasing the subsequent risk of overcorrection (5).

PP is closely related to schizophrenia, although it can occur in other psychiatric conditions including affective disorders, anorexia nervosa and substance abuse disorders (6). The most common complication of PP is hyponatraemia. Under normal physiological conditions, excessive fluid intake results in a decrease in serum osmolality and inhibition of AVP release leading to hypotonic polyuria. In these circumstances, the excretory capacity of the kidneys can increase up to 15-18 L/day (7). Increased urine output will compensate for excessive fluid intake, meaning that the majority of patients with PP remain eunatraemic. In SIAD, the dilution capacity of the kidneys is impaired, significantly reducing the amount of free fluid intake necessary to develop hyponatraemia. Recently, a prospective cohort study reported that all patients with PP, admitted with profound hyponatraemia $(<125 \mathrm{mmol} / \mathrm{L})$ exhibited impaired water secretion. All patients received at least one psychotropic medication that was known to cause SIAD. Other nonosmotic stimuli including nausea and infection occurred with incidences of 43 and 14\%, respectively (6). Acute psychosis is another potential stimulus of SIAD in patients with schizophrenia. We thought this was unlikely in our case as the profound hyponatraemia occurred 5 weeks after the initial presentation, and the patient was improving from a psychiatric perspective. Evidence on the long-term complications of PP is limited. However, one study reported that $66 \%$ of patients were readmitted within 1 year, and $50 \%$ of patients had a subsequent episode of hyponatraemia (6). The mortality rate in patients with PP and schizophrenia was higher than those with schizophrenia alone. In another retrospective observational study, (8) the median age of death in those with PP and schizophrenia was 59 years compared with 68 years in those with schizophrenia alone.

Hyponatraemia, itself, is a well-documented side effect of many psychotropic medications including selective serotonin reuptake inhibitors (SSRIs) and first-generation anti-psychotics. SGAs can also induce hyponatraemia either through the non-osmotic stimulus of AVP or enhancing the activity of AVP at the kidneys. The incidence of hyponatraemia after commencing SGAs remains unknown, although it is likely under-reported given the contaminant prescription of other psychotropic medications. In one population-based cohort study, the 30-day hospitilisation rate for hyponatraemia after commencing SGAs was $0.09 \%$ which was $50 \%$ higher than those not on SGAs (9). In a case-control study using an international safety monitoring database, the risk of hyponatraemia was increased to 1.5 -fold in patients prescribed to SGAs relative to controls (10). This study identified olanzapine, risperidone and clozapine amongst the SGAs most commonly associated with hyponatraemia. A major limitation of this study, however, was that the dosages of each SGAs were not taken into account. Risk factors for SGA-induced hyponatraemia include increasing age, female sex and co-prescription of SSRIs and thiazide diuretics. Hyponatraemia most commonly occurs with the first 2 weeks after commencing antipsychotics but can also occur after longer-term use. Current guidelines on the management of schizophrenia do not address monitoring for hyponatremia when commencing SGAs. It is our recommendation to check $\mathrm{Na}^{+}$levels one to two times within the first 2 weeks of commencing or adjusting a SGA. More frequent monitoring may be required in individuals with pre-existing hyponatraemia and risk factors for SGAsinduced hyponatraemia. Particular care must be taken in individuals with PP as the combination of excessive fluid intake and SGA-induced SIAD can cause a life-threatening hyponatraemia as outlined in this case. Given the widespread use of SGAs, systematic studies are required to quantify the risk of hyponatraemia and support evidencebased guidelines.

Patients with PP and SIAD are at high risk of sodium overcorrection if they are fluid restricted or the stimulus for AVP is removed. In this case, it occurred with holding quetiapine. ODS is a devastating neurological condition that can occur from a rapid correction in serum $\mathrm{Na}^{+}$. It can manifest with a spastic quadriparesis, cranial nerve palsies, pseudobulbar palsy or locked in syndrome. Patients with a sodium of less than $105 \mathrm{mmol} / \mathrm{L}$ are at increased risk ODS (11). Guidelines on the management of hyponatraemia recommend a maximum increase in sodium of $8 \mathrm{mmol} / \mathrm{L}$ over $24 \mathrm{~h}$ for those at high risk of ODS (1). Hourly urine output and frequent serum $\mathrm{Na}^{+}$ measurements are required to promptly identify patients whose $\mathrm{Na}^{+}$is at risk of or has overcorrected. Treatment of 
$\mathrm{Na}^{+}$overcorrection requires administration of either $5 \%$ dextrose to replace free water losses or desmopressin, an AVP analogue.

This case highlights that the combination of $\mathrm{PP}$ and SIAD can result in life-threatening extreme hyponatraemia. In patients with $\mathrm{PP}$ who present with altered behaviour, confusion or vomiting, symptomatic hyponatraemia should be included in the differential diagnosis. A low threshold of suspicion is required to promptly diagnose this condition and instigate emergency treatment with boluses of hypertonic $3 \%$ saline. Second-generation antipsychotics are an uncommon but important cause of SIAD in patients with schizophrenia and PP. When commencing or adjusting antipsychotic medications, especially in those with PP, monitoring for hyponatraemia should be undertaken. Further systematic studies are required to quantify the risk of hyponatraemia associated with secondgeneration guidelines to facilitate the development of evidence-based guidelines.

\section{Declaration of interest}

The authors declare that there is no conflict of interest that could be perceived as prejudicing the impartiality of the research reported.

\section{Funding}

This work did not receive any specific grant from any funding agency in the public, commercial, or not-for-profit sector.

\section{Patient consent}

The patient has provided written consent for publication of this case.

\section{Author contribution statement}

All authors were involved in the clinical care of the patient.

\section{References}

1 Spasovski G, Vanholder R, Allolio B, Annane D, Ball S, Bichet D, Decaux G, Fenske W, Hoorn EJ, Ichai C, et al. Clinical practice guideline on diagnosis and treatment of hyponatraemia. European Journal of Endocrinology 2014 170 G1-G47. (https://doi.org/10.1530/EJE-13-1020)

2 Renneboog B, Musch W, Vandemergel X, Manto MU \& Decaux G. Mild chronic hyponatremia is associated with falls, unsteadiness, and attention deficits. American Journal of Medicine 2006119 71.e1-71.e8. (https://doi.org/10.1016/j.amjmed.2005.09.026)

3 Mercier-Guidez E \& Loas G. Polydipsia and water intoxication in 353 psychiatric inpatients: an epidemiological and psychopathological study. European Psychiatry 200015 306-311. (https://doi.org/10.1016/ s0924-9338(00)00399-0)

4 Verbalis JG, Goldsmith SR, Greenberg A, Korzelius C, Schrier RW, Sterns RH \& Thompson CJ. Diagnosis, evaluation, and treatment of hyponatremia: expert panel recommendations. American Journal of Medicine 2013126 (Supplement 1) S1-S42. (https://doi.org/10.1016/j. amjmed.2013.07.006)

5 Garrahy A, Dineen R, Hannon AM, Cuesta M, Tormey W, Sherlock M $\&$ Thompson CJ. Continuous versus bolus infusion of hypertonic saline in the treatment of symptomatic hyponatremia caused by SIAD. Journal of Clinical Endocrinology and Metabolism 2019104 3595-3602. (https://doi.org/10.1210/jc.2019-00044)

6 Sailer CO, Winzeler B, Nigro N, Suter-Widmer I, Arici B, Bally M, Schuetz P, Mueller B \& Christ-Crain M. Characteristics and outcomes of patients with profound hyponatraemia due to primary polydipsia. Clinical Endocrinology 201787 492-499. (https://doi.org/10.1111/cen.13384)

7 Berl T. Impact of solute intake on urine flow and water excretion. Journal of the American Society of Nephrology 200819 1076-1078.

8 Hawken ER, Crookall JM, Reddick D, Millson RC, Milev R \& Delva N. Mortality over a 20-year period in patients with primary polydipsia associated with schizophrenia: a retrospective study. Schizophrenia Research 2009107 128-133. (https://doi.org/10.1016/j. schres.2008.09.029)

9 Gandhi S, McArthur E, Reiss JP, Mamdani MM, Hackam DG, Weir MA \& Garg AX. Atypical antipsychotic medications and hyponatremia in older adults: a population-based cohort study. Canadian Journal of Kidney Health and Disease 20163 21. (https://doi.org/10.1186/s40697-016-0111-z)

10 Mannesse CK, Van Puijenbroek EP, Jansen PA, Van Marum RJ, Souverein PC \& Egberts TC. Hyponatraemia as an adverse drug reaction of antipsychotic drugs: a case-control study in VigiBase. Drug Safety 2010 33 569-578. (https://doi.org/10.2165/11532560-000000000-00000)

$11 \mathrm{Vu}$ T, Wong R, Hamblin PS, Zajac J \& Grossmann MJHP. Patients presenting with severe hypotonic hyponatremia: etiological factors, assessment, and outcomes. Hospital Practice 200937 128-136. (https:// doi.org/10.3810/hp.2009.12.266)

Received in final form 31 July 2021

Accepted 6 September 2021 